\title{
The Strengthening the Reporting of Observational Studies in Epidemiology (STROBE) Statement: Guidelines for Reporting Observational Studies
}

\author{
Erik von Elm ${ }^{1 *}$, Douglas G. Altman ${ }^{2}$, Matthias Egger ${ }^{1,3}$, Stuart J. Pocock ${ }^{4}$, Peter C. Gøtzsche ${ }^{5}$, \\ Jan P. Vandenbroucke ${ }^{6}$ for the STROBE Initiative \\ 1 Institute of Social and Preventive Medicine (ISPM), University of Bern, Bern, Switzerland, 2 Centre for Statistics in Medicine, University of Oxford, Oxford, United Kingdom, \\ 3 Department of Social Medicine, University of Bristol, Bristol, United Kingdom, 4 London School of Hygiene and Tropical Medicine, University of London, London, United \\ Kingdom, 5 Nordic Cochrane Centre, Copenhagen, Denmark, 6 Department of Clinical Epidemiology, Leiden University Hospital, Leiden, The Netherlands
}

Funding: The workshop was funded by the European Science Foundation (ESF). Additional funding was received from the Medical Research Council Health Services Research Collaboration and the National Health Services Research and Development Methodology Programme. The funders had no role in study design, data collection and analysis, decision to publish, or preparation of the manuscript.

Competing Interests: The authors have declared that no competing interests exist.

Citation: von Elm E, Altman DG, Egger M, Pocock SJ, Gøtzsche PC, et al. (2007) The Strengthening the Reporting of Observational Studies in Epidemiology (STROBE) Statement: Guidelines for reporting observational studies. PLoS Med 4(10): e296. 10.1371/journal.pmed. 0040296

Received: June 20, 2007

Accepted: August 30, 2007

Published: October 16, 2007

Copyright: (c) 2007 von Elm et al. This is an open-access article distributed under the terms of the Creative Commons Attribution License, which permits unrestricted use, distribution, and reproduction in any medium provided the original author and source are credited. For details on further use, see the STROBE Web site (http://www.strobe-statement.org/). In order to encourage dissemination of the STROBE Statement, this article will also be published and made freely available by Annals of Internal Medicine, BMJ Bulletin of the World Health Organization, Epidemiology, The Lancet, and Preventive Medicine.

Abbreviations: CONSORT

Consolidated Standards of Reporting Trials; STREGA, STROBE Extension to Genetic Association Studies; STROBE, Strengthening the Reporting of Observational Studies in

Epidemiology

* To whom correspondence should be addressed. E-mail: strobe@ispm. unibe.ch

\section{A B S T R A C T}

Much biomedical research is observational. The reporting of such research is often inadequate, which hampers the assessment of its strengths and weaknesses and of a study's generalisability. The Strengthening the Reporting of Observational Studies in Epidemiology (STROBE) Initiative developed recommendations on what should be included in an accurate and complete report of an observational study. We defined the scope of the recommendations to cover three main study designs: cohort, case-control, and cross-sectional studies. We convened a 2-day workshop in September 2004, with methodologists, researchers, and journal editors to draft a checklist of items. This list was subsequently revised during several meetings of the coordinating group and in e-mail discussions with the larger group of STROBE contributors, taking into account empirical evidence and methodological considerations. The workshop and the subsequent iterative process of consultation and revision resulted in a checklist of 22 items (the STROBE Statement) that relate to the title, abstract, introduction, methods, results, and discussion sections of articles. 18 items are common to all three study designs and four are specific for cohort, case-control, or cross-sectional studies. A detailed Explanation and Elaboration document is published separately and is freely available on the Web sites of PLoS Medicine, Annals of Internal Medicine, and Epidemiology. We hope that the STROBE Statement will contribute to improving the quality of reporting of observational studies. 


\section{Introduction}

Many questions in medical research are investigated in observational studies [1]. Much of the research into the cause of diseases relies on cohort, case-control, or cross-sectional studies. Observational studies also have a role in research into the benefits and harms of medical interventions [2]. Randomised trials cannot answer all important questions about a given intervention. For example, observational studies are more suitable to detect rare or late adverse effects of treatments, and are more likely to provide an indication of what is achieved in daily medical practice [3].

Research should be reported transparently so that readers can follow what was planned, what was done, what was found, and what conclusions were drawn. The credibility of research depends on a critical assessment by others of the strengths and weaknesses in study design, conduct, and analysis. Transparent reporting is also needed to judge whether and how results can be included in systematic reviews [4,5]. However, in published observational research important information is often missing or unclear. An analysis of epidemiological studies published in general medical and specialist journals found that the rationale behind the choice of potential confounding variables was often not reported [6]. Only few reports of case-control studies in psychiatry explained the methods used to identify cases and controls [7]. In a survey of longitudinal studies in stroke research, 17 of 49 articles $(35 \%)$ did not specify the eligibility criteria [8]. Others have argued that without sufficient clarity of reporting, the benefits of research might be achieved more slowly [9], and that there is a need for guidance in reporting observational studies [10,11].

Recommendations on the reporting of research can improve reporting quality. The Consolidated Standards of Reporting Trials (CONSORT) Statement was developed in 1996 and revised 5 years later [12]. Many medical journals supported this initiative [13], which has helped to improve the quality of reports of randomised trials [14,15]. Similar initiatives have followed for other research areas-e.g., for the reporting of meta-analyses of randomised trials [16] or diagnostic studies [17]. We established a network of methodologists, researchers, and journal editors to develop recommendations for the reporting of observational research: the Strengthening the Reporting of Observational Studies in Epidemiology (STROBE) Statement.

\section{Aims and Use of the STROBE Statement}

The STROBE Statement is a checklist of items that should be addressed in articles reporting on the 3 main study designs of analytical epidemiology: cohort, case-control, and crosssectional studies. The intention is solely to provide guidance on how to report observational research well: these recommendations are not prescriptions for designing or conducting studies. Also, while clarity of reporting is a prerequisite to evaluation, the checklist is not an instrument to evaluate the quality of observational research.

Here we present the STROBE Statement and explain how it was developed. In a detailed companion paper, the Explanation and Elaboration article [18-20], we justify the inclusion of the different checklist items and give methodological background and published examples of what we consider transparent reporting. We strongly recommend using the STROBE checklist in conjunction with the explanatory article, which is available freely on the Web sites of PLoS Medicine (http://www.plosmedicine.org/), Annals of Internal Medicine (http://www.annals.org/), and Epidemiology (http:// www.epidem.com/).

\section{Development of the STROBE Statement}

We established the STROBE Initiative in 2004, obtained funding for a workshop and set up a Web site (http://www. strobe-statement.org/). We searched textbooks, bibliographic databases, reference lists, and personal files for relevant material, including previous recommendations, empirical studies of reporting and articles describing relevant methodological research. Because observational research makes use of many different study designs, we felt that the scope of STROBE had to be clearly defined early on. We decided to focus on the 3 study designs that are used most widely in analytical observational research: cohort, case-control, and cross-sectional studies.

We organised a 2-day workshop in Bristol, UK, in September 2004. 23 individuals attended this meeting, including editorial staff from Annals of Internal Medicine, $B M J$, Bulletin of the World Health Organization, International Journal of Epidemiology, JAMA, Preventive Medicine, and The Lancet, as well as epidemiologists, methodologists, statisticians, and practitioners from Europe and North America. Written contributions were sought from 10 other individuals who declared an interest in contributing to STROBE, but could not attend. Three working groups identified items deemed to be important to include in checklists for each type of study. A provisional list of items prepared in advance (available from our Web site) was used to facilitate discussions. The 3 draft checklists were then discussed by all participants and, where possible, items were revised to make them applicable to all three study designs. In a final plenary session, the group decided on the strategy for finalizing and disseminating the STROBE Statement.

After the workshop we drafted a combined checklist including all three designs and made it available on our Web site. We invited participants and additional scientists and editors to comment on this draft checklist. We subsequently published 3 revisions on the Web site, and 2 summaries of comments received and changes made. During this process the coordinating group (i.e., the authors of the present paper) met on eight occasions for 1 or 2 days and held several telephone conferences to revise the checklist and to prepare the present paper and the Explanation and Elaboration paper [18-20]. The coordinating group invited 3 additional co-authors with methodological and editorial expertise to help write the Explanation and Elaboration paper, and sought feedback from more than 30 people, who are listed at the end of this paper. We allowed several weeks for comments on subsequent drafts of the paper and reminded collaborators about deadlines by e-mail.

\section{STROBE Components}

The STROBE Statement is a checklist of 22 items that we consider essential for good reporting of observational studies (Table 1). These items relate to the article's title and abstract 
Table 1. The STROBE Statement-Checklist of Items That Should Be Addressed in Reports of Observational Studies

Item

number

\section{Recommendation}

TITLE and ABSTRACT 11 (a) Indicate the study's design with a commonly used term in the title or the abstract

(b) Provide in the abstract an informative and balanced summary of what was done and what was found

INTRODUCTION

Background/

rationale

Objectives
2 Explain the scientific background and rationale for the investigation being reported

3 State specific objectives, including any prespecified hypotheses

\section{METHODS}

Study design

Setting

Participants

Present key elements of study design early in the paper

Describe the setting, locations, and relevant dates, including periods of recruitment, exposure, follow-up, and data collection

(a) Cohort study-Give the eligibility criteria, and the sources and methods of selection of participants. Describe methods of follow-up

Case-control study-Give the eligibility criteria, and the sources and methods of case ascertainment and control selection. Give the rationale for the choice of cases and controls

Cross-sectional study-Give the eligibility criteria, and the sources and methods of selection of participants

(b) Cohort study-For matched studies, give matching criteria and number of exposed and unexposed Case-control study-For matched studies, give matching criteria and the number of controls per case

Variables

Data sources/

measurement

Bias

Study size

Quantitative

variables

Statistical

methods
7

Clearly define all outcomes, exposures, predictors, potential confounders, and effect modifiers. Give diagnostic criteria, if applicable

For each variable of interest, give sources of data and details of methods of assessment (measurement).

Describe comparability of assessment methods if there is more than one group

Describe any efforts to address potential sources of bias

Explain how the study size was arrived at

Explain how quantitative variables were handled in the analyses. If applicable, describe which groupings were chosen, and why

12 (a) Describe all statistical methods, including those used to control for confounding

(b) Describe any methods used to examine subgroups and interactions

(c) Explain how missing data were addressed

(d) Cohort study-If applicable, explain how loss to follow-up was addressed

Case-control study-If applicable, explain how matching of cases and controls was addressed

Cross-sectional study - If applicable, describe analytical methods taking account of sampling strategy

(e) Describe any sensitivity analyses

\section{RESULTS}

Participants

(a) Report the numbers of individuals at each stage of the study-e.g., numbers potentially eligible, examined for eligibility, confirmed eligible, included in the study, completing follow-up, and analysed

(b) Give reasons for non-participation at each stage

(c) Consider use of a flow diagram

Descriptive

data

$14^{*}$

(a) Give characteristics of study participants (e.g., demographic, clinical, social) and information on exposures and potential confounders

(b) Indicate the number of participants with missing data for each variable of interest

(c) Cohort study - Summarise follow-up time (e.g., average and total amount)

Outcome data $\quad 15^{*} \quad$ Cohort study-Report numbers of outcome events or summary measures over time

Case-control study-Report numbers in each exposure category, or summary measures of exposure

Cross-sectional study-Report numbers of outcome events or summary measures

Main results

16 (a) Give unadjusted estimates and, if applicable, confounder-adjusted estimates

Make clear which confounders were adjusted for and why they were included

(b) Report category boundaries when continuous variables were categorized

(c) If relevant, consider translating estimates of relative risk into absolute risk for a meaningful time period

Other

analyses

Report other analyses done-e.g., analyses of subgroups and interactions, and sensitivity analyses
DISCUSSION

Key results

Limitations

Interpretation

Generalisability
Summarise key results with reference to study objectives

Discuss limitations of the study, taking into account sources of potential bias or imprecision. Discuss both direction and magnitude of any potential bias

Give a cautious overall interpretation of results considering objectives, limitations, multiplicity of analyses, results from similar studies, and other relevant evidence

Discuss the generalisability (external validity) of the study results

OTHER INFORMATION

Funding

Give the source of funding and the role of the funders for the present study and, if applicable, for the original study on which the present article is based

*Give such information separately for cases and controls in case-control studies, and, if applicable, for exposed and unexposed groups in cohort and cross-sectional studies.
Note: An Explanation and Elaboration article discusses each checklist item and gives methodological background and published examples of transparent reporting. The STROBE checklist Note: An Explanation and Elaboration article discusses each checklist item and gives methodological background and published examples of transparent reporting. The STROBE checklist
is best used in conjunction with this article (freely available on the Web sites of PLoS Medicine at http://www.plosmedicine.org/, Annals of Internal Medicine at http://www.annals.org/, and is best used in conjunction with this article (freely available on the Web sites of PLoS Medicine at http://www.plosmedicine.org/, Annals of Internal Medicine at http://www.annals.org/, and
Epidemiology at http://www.epidem.com/). Separate versions of the checklist for cohort, case-control, and cross-sectional studies are available on the STROBE Web site at http://www.

doi:10.1371/journal.pmed.0040296.t001

PLoS Medicine I www.plosmedicine.org 
(item 1), the introduction (items 2 and 3), methods (items 4 12), results (items 13-17) and discussion sections (items 1821), and other information (item 22 on funding). 18 items are common to all three designs, while four (items $6,12,14$, and 15) are design-specific, with different versions for all or part of the item. For some items (indicated by asterisks), information should be given separately for cases and controls in case-control studies, or exposed and unexposed groups in cohort and cross-sectional studies. Although presented here as a single checklist, separate checklists are available for each of the 3 study designs on the STROBE Web site.

\section{Implications and Limitations}

The STROBE Statement was developed to assist authors when writing up analytical observational studies, to support editors and reviewers when considering such articles for publication, and to help readers when critically appraising published articles. We developed the checklist through an open process, taking into account the experience gained with previous initiatives, in particular CONSORT. We reviewed the relevant empirical evidence as well as methodological work, and subjected consecutive drafts to an extensive iterative process of consultation. The checklist presented here is thus based on input from a large number of individuals with diverse backgrounds and perspectives. The comprehensive explanatory article [18-20], which is intended for use alongside the checklist, also benefited greatly from this consultation process.

Observational studies serve a wide range of purposes, on a continuum from the discovery of new findings to the confirmation or refutation of previous findings [18-20]. Some studies are essentially exploratory and raise interesting hypotheses. Others pursue clearly defined hypotheses in available data. In yet another type of studies, the collection of new data is planned carefully on the basis of an existing hypothesis. We believe the present checklist can be useful for all these studies, since the readers always need to know what was planned (and what was not), what was done, what was found, and what the results mean. We acknowledge that STROBE is currently limited to three main observational study designs. We would welcome extensions that adapt the checklist to other designs-e.g., case-crossover studies or ecological studies-and also to specific topic areas. Four extensions are now available for the CONSORT statement [21-24]. A first extension to STROBE is underway for genedisease association studies: the STROBE Extension to Genetic Association studies (STREGA) initiative [25]. We ask those who aim to develop extensions of the STROBE Statement to contact the coordinating group first to avoid duplication of effort.

The STROBE Statement should not be interpreted as an attempt to prescribe the reporting of observational research in a rigid format. The checklist items should be addressed in sufficient detail and with clarity somewhere in an article, but the order and format for presenting information depends on author preferences, journal style, and the traditions of the research field. For instance, we discuss the reporting of results under a number of separate items, while recognizing that authors might address several items within a single section of text or in a table. Also, item 22, on the source of funding and the role of funders, could be addressed in an appendix or in the methods section of the article. We do not aim at standardising reporting. Authors of randomised clinical trials were asked by an editor of a specialist medical journal to "CONSORT" their manuscripts on submission [26]. We believe that manuscripts should not be "STROBEd", in the sense of regulating style or terminology. We encourage authors to use narrative elements, including the description of illustrative cases, to complement the essential information about their study, and to make their articles an interesting read [27].

We emphasise that the STROBE Statement was not developed as a tool for assessing the quality of published observational research. Such instruments have been developed by other groups and were the subject of a recent systematic review [28]. In the Explanation and Elaboration paper, we used several examples of good reporting from studies whose results were not confirmed in further research - the important feature was the good reporting, not whether the research was of good quality. However, if STROBE is adopted by authors and journals, issues such as confounding, bias, and generalisability could become more transparent, which might help temper the over-enthusiastic reporting of new findings in the scientific community and popular media [29], and improve the methodology of studies in the long term. Better reporting may also help to have more informed decisions about when new studies are needed, and what they should address.

We did not undertake a comprehensive systematic review for each of the checklist items and sub-items, or do our own research to fill gaps in the evidence base. Further, although no one was excluded from the process, the composition of the group of contributors was influenced by existing networks and was not representative in terms of geography (it was dominated by contributors from Europe and North America) and probably was not representative in terms of research interests and disciplines. We stress that STROBE and other recommendations on the reporting of research should be seen as evolving documents that require continual assessment, refinement, and, if necessary, change. We welcome suggestions for the further dissemination of STROBE-e.g., by re-publication of the present article in specialist journals and in journals published in other languages. Groups or individuals who intend to translate the checklist to other languages should consult the coordinating group beforehand. We will revise the checklist in the future, taking into account comments, criticism, new evidence, and experience from its use. We invite readers to submit their comments via the STROBE Web site (http://www.strobe-statement.org/).

\section{Acknowledgments}

We are grateful to Gerd Antes, Kay Dickersin, Shah Ebrahim, and Richard Lilford for supporting the STROBE Initiative. We are grateful to the following institutions that have hosted working meetings of the coordinating group: Institute of Social and Preventive Medicine (ISPM), University of Bern, Bern, Switzerland; Department of Social Medicine, University of Bristol, Bristol, UK; London School of Hygiene and Tropical Medicine, London, UK; Nordic Cochrane Centre, Copenhagen, Denmark; and Centre for Statistics in Medicine, Oxford, UK. We are grateful to six reviewers who provided helpful comments on a previous draft of this paper.

\section{Contributors to the STROBE Initiative}

The following individuals have contributed to the content and elaboration of the STROBE Statement: Douglas G Altman, Maria Blettner, Paolo Boffetta, Hermann Brenner, Geneviève Chêne, Cyrus 
Cooper, George Davey-Smith, Erik von Elm, Matthias Egger, France Gagnon, Peter C Gøtzsche, Philip Greenland, Sander Greenland, Claire Infante-Rivard, John Ioannidis, Astrid James, Giselle Jones, Bruno Ledergerber, Julian Little, Margaret May, David Moher, Hooman Momen, Alfredo Morabia, Hal Morgenstern, Cynthia D Mulrow, Fred Paccaud, Stuart J Pocock, Charles Poole, Martin Röösli, Dietrich Rothenbacher, Kenneth Rothman, Caroline Sabin, Willi Sauerbrei, Lale Say, James J Schlesselman, Jonathan Sterne, Holly Syddall, Jan P Vandenbroucke, Ian White, Susan Wieland, Hywel Williams, Guang Yong Zou.

Author contributions. The authors coordinated the STROBE Initiative and contributed to the writing of the paper. EvE wrote the first draft of the paper and takes care of most of the practical coordination of STROBE. ME initiated STROBE and, together with EvE, organised the first workshop.

\section{References}

1. Glasziou P, Vandenbroucke JP, Chalmers I (2004) Assessing the quality of research. BMJ 328: 39-41.

2. Black N (1996) Why we need observational studies to evaluate the effectiveness of health care. BMJ 312: 1215-1218

3. Papanikolaou PN, Christidi GD, Ioannidis JP (2006) Comparison of evidence on harms of medical interventions in randomized and nonrandomized studies. CMAJ 174: 635-641.

4. Jüni P, Altman DG, Egger M (2001) Systematic reviews in health care: Assessing the quality of controlled clinical trials. BMJ 323: 42-46.

5. Egger M, Schneider M, Davey Smith G (1998) Spurious precision? Metaanalysis of observational studies. BMJ 316: 140-144.

6. Pocock SJ, Collier TJ, Dandreo KJ, de Stavola BL, Goldman MB, et al. (2004) Issues in the reporting of epidemiological studies: a survey of recent practice. BMJ 329: 883 .

7. Lee W, Bindman J, Ford T, Glozier N, Moran P, et al. (2007) Bias in psychiatric case-control studies: literature survey. Br J Psychiatry 190: 204209.

8. Tooth L, Ware R, Bain C, Purdie DM, Dobson A (2005) Quality of reporting of observational longitudinal research. Am J Epidemiol 161: 280-288.

9. Bogardus ST Jr., Concato J, Feinstein AR (1999) Clinical epidemiological quality in molecular genetic research: the need for methodological standards. JAMA 281: 1919-1926.

10. Anonymous (1981) Guidelines for documentation of epidemiologic studies. Epidemiology Work Group of the Interagency Regulatory Liaison Group. Am J Epidemiol 114: 609-613.

11. Rennie D (2001) CONSORT revised-improving the reporting of randomized trials. JAMA. 285: 2006-2007.

12. Moher D, Schulz KF, Altman DG (2001) The CONSORT statement: revised recommendations for improving the quality of reports of parallel-group randomised trials. Lancet 357: 1191-1194.

13. Moher D, Altman DG, Schulz KF, Elbourne DR (2004) Opportunities and challenges for improving the quality of reporting clinical research: CONSORT and beyond. CMAJ 171: 349-350.

14. Plint AC, Moher D, Morrison A, Schulz K, Altman DG, et al. (2006) Does the CONSORT checklist improve the quality of reports of randomised controlled trials? A systematic review. Med J Aust 185: 263-267.

15. Egger M, Jüni P, Bartlett C (2001) Value of flow diagrams in reports of randomized controlled trials. JAMA 285: 1996-1999.

16. Moher D, Cook DJ, Eastwood S, Olkin I, Rennie D, et al. (1999) Improving the quality of reports of meta-analyses of randomised controlled trials: the QUOROM statement. Quality of Reporting of Meta-analyses. Lancet 354: $1896-1900$.

17. Bossuyt PM, Reitsma JB, Bruns DE, Gatsonis CA, Glasziou PP, et al. (2003) Towards complete and accurate reporting of studies of diagnostic accuracy: The STARD Initiative. Ann Intern Med. 138: 40-44.

18. Vandenbroucke JP, von Elm E, Altman DG, Gøtzsche PC, Mulrow CD, et al. for the STROBE Initiative (2007) Strengthening the Reporting of Observational Studies in Epidemiology (STROBE): Explanation and Elaboration. PLoS Med 4: e297. doi:10.1371/journal.pmed.0040297

19. Vandenbroucke JP, von Elm E, Altman DG, Gøtzsche PC, Mulrow CD, et al. for the STROBE Initiative (2007) Strengthening the Reporting of Observational Studies in Epidemiology (STROBE): Explanation and Elaboration. Ann Intern Med. In press.

20. Vandenbroucke JP, von Elm E, Altman DG, Gøtzsche PC, Mulrow CD, et al. for the STROBE Initiative (2007) Strengthening the Reporting of Observational Studies in Epidemiology (STROBE): Explanation and Elaboration. Epidemiology. In press.

21. Ioannidis JP, Evans SJ, Gøtzsche PC, O'Neill RT, Altman DG, et al. (2004) Better reporting of harms in randomized trials: an extension of the CONSORT statement. Ann Intern Med 141: 781-788.

22. Campbell MK, Elbourne DR, Altman DG (2004) CONSORT statement: extension to cluster randomised trials. BMJ 328: 702-708

23. Piaggio G, Elbourne DR, Altman DG, Pocock SJ, Evans SJ (2006) Reporting of noninferiority and equivalence randomized trials: an extension of the CONSORT statement. JAMA 295: 1152-1160.

24. Gagnier JJ, Boon H, Rochon P, Moher D, Barnes J, et al. (2006) Reporting randomized, controlled trials of herbal interventions: an elaborated CONSORT statement. Ann Intern Med 144: 364-367.

25. Ioannidis JP, Gwinn M, Little J, Higgins JP, Bernstein JL, et al. (2006) A road map for efficient and reliable human genome epidemiology. Nat Genet 38: $3-5$.

26. Ormerod AD (2001) CONSORT your submissions: an update for authors. Br J Dermatol 145: 378-379.

27. Schriger DL (2005) Suggestions for improving the reporting of clinical research: the role of narrative. Ann Emerg Med 45: 437-443.

28. Sanderson S, Tatt ID, Higgins JP (2007) Tools for assessing quality and susceptibility to bias in observational studies in epidemiology: a systematic review and annotated bibliography. Int J Epidemiol 36: 666-676.

29. Bartlett C, Sterne J, Egger M (2002) What is newsworthy? Longitudinal study of the reporting of medical research in two British newspapers. BMJ 325: 81-84. 